\title{
Seasonal and solar cycle variations in the ionospheric convection reversal boundary location inferred from monthly SuperDARN data sets
}

\author{
Alexander V. Koustov ${ }^{1}$ and Robyn A. D. Fiori ${ }^{2}$ \\ ${ }^{1}$ Institute of Space and Atmospheric Studies, University of Saskatchewan, Saskatoon, Canada \\ ${ }^{2}$ Geomagnetic Laboratory, Natural Resources Canada, Ottawa, Canada \\ Correspondence to: Alexander V. Koustov (sasha.koustov@usask.ca) \\ Received: 7 August 2015 - Revised: 14 January 2016 - Accepted: 29 January 2016 - Published: 15 February 2016
}

\begin{abstract}
Multi-year (1995-2013) velocity data collected by the Super Dual Auroral Network (SuperDARN) HF radars are considered to investigate seasonal and solar cycle variations of the convection reversal boundary (CRB) location for interplanetary magnetic field (IMF) $B_{z}<0$. By considering monthly data sets we show that the CRB is at higher latitudes in summer between 1995 and 2007. The poleward shifts are on the order of $2-5^{\circ}$. After 2007, the seasonal effect weakens, and the highest latitudes for the CRB start to occur during the winter time. We show that the CRB latitudes decrease with an increase of the IMF transverse component at a rate of $\left(1-2^{\circ}\right) / 2 n T$. Because of this effect, on average, the $\mathrm{CRB}$ latitudes are lower during high solar activity periods with stronger IMFs. We also confirm the effect of the CRB dawn-dusk shifts related to the IMF changes in the IMF $B_{y}$ sign.
\end{abstract}

Keywords. Ionosphere (plasma convection)

\section{Introduction}

Ground-based and satellite-based observations carried over the last 3 decades have converged to a firm understanding that the global-scale ionospheric plasma circulation follows a two-celled pattern for southward-oriented interplanetary magnetic field (IMF) $B_{z}\left(B_{z}<0\right)$. The list of relevant publications is extensive; it includes statistical models based on individual instruments (e.g., Foster, 1983; Ruohoniemi and Greenwald, 1996, 2005; Papitashvili and Rich, 2002) and instantaneous mapping involving multiple instruments (e.g., Knipp et al., 1993; Lu et al., 1994). In a two-celled pattern, the plasma flows away from noon across the central polar cap and, after reaching the nightside at auroral oval latitudes, deviates to dawn and dusk and starts moving backward, towards noon, at lower magnetic latitudes. Another reverse in the direction of the flow happens near noon, on the dusk and dawn sides, so that each flow cell becomes a closed loop. The shape of the convection cells and intensity of the flow depends on several factors; the magnitude and orientation of the IMF are believed to be the most important ones (e.g., Zhang et al., 2007 and references therein). While the IMF $B_{z}$ affects the size of the cells (influencing the rate of front side magnetic flux line merging, e.g. Cowley and Lockwood, 1992), the IMF $B_{y}$ affects their shape. In the Northern Hemisphere, for positive IMF $B_{y}$, the dusk cell is more round and larger in size than the dawn cell which has a crescent shape. The shape and size of the convection cells reverses for negative IMF $B_{y}$. The asymmetry of the cells becomes progressively stronger as the magnitude of $B_{y}$ increases. The transpolar flow over the Magnetic Pole is usually not aligned with the noon-midnight meridian; it is "rotated" around the Pole to prenoon for $B_{y}>0$ and to postnoon for $B_{y}<0$. With the advance of season from winter to summer, the misalignment increases (Zhang et al., 2007). An important factor affecting the shape of the convection cells is the conductance at high latitudes and its global-scale distribution (e.g., Zhang et al., 2007).

An important characteristic of a two-celled ionospheric plasma flow pattern is the convection reversal boundary $(\mathrm{CRB})$ that can be introduced as a curve, on a magnetic local time-magnetic latitude (MLT-MLAT) plane, that delineates anti-sunward plasma flow across the polar cap and sun- 
ward flow at auroral oval latitudes (e.g., Ridley and Clauer, 1996). The CRB location is easier to recognize at dawn and dusk where the ionospheric plasma flows predominantly along the magnetic parallels and the flow shear is prominent. However, the pattern is often so round and/or circular, especially near noon and midnight hours (Ridley and Clauer, 1996), that the CRB quantification, as a separator of regions with opposing flows, is difficult. The CRB is located closely to an ionospheric projection of the open and/or closed magnetic field lines boundary (e.g., Sotirelis et al., 2005), and for this reason it is of particular interest. Hubert et al. (2010) compared the location of several proxies for the open-closed field lines boundary, including the $\mathrm{CRB}$, and concluded that as a proxy for the open-closed boundary, the CRB is not as reliable in the midnight sector, near the Harang discontinuity.

Statistical studies by de la Beaujardiere et al. (1991) and Bristow and Spaleta (2013) showed that the CRB is a circlelike line squeezed toward higher latitudes on the dayside and toward lower latitudes on the nightside. The size of this deformed circle and its shape depend on the IMF $B_{z}$ and $B_{y}$. One known effect is the CRB shift to lower latitude with a typical rate of $2^{\circ} / 3 \mathrm{nT}$ when the IMF $B_{z}$ becomes more negative (Bristow and Spaleta, 2013). The shift is stronger on the dayside (Chen et al., 2015). The effect of the IMF $B_{y}$ is more complicated. With an increase of positive $B_{y}$, the CRB on the dusk side shifts to higher latitudes whereas with an increase in negative $B_{y}$ (to more negative values), the CRB on the dusk side shifts to lower latitudes (Bristow and Spaleta, 2013; Chen et al., 2015). The $B_{y}$ effect can be envisioned as a shift of the CRB "circle" as a whole toward dawn for $B_{y}>0$ and toward dusk for $B_{y}<0$. On average, the CRB shifts are on the order of $2-3^{\circ}$ which is not a very strong effect. It is not a surprise that the CRB is often fairly stable although latitudinal motions and even periodic undulations are possible (Ridley and Clauer, 1996).

For identification of the location of the CRB, a 2-D flow pattern on a large scale has to be known. In the past, the patterns have been studied with magnetometers (e.g., Papitashvili et al., 1994), radars (e.g., Ridley and Clauer, 1996; Rash et al., 1999) and electric field sensors on satellites (e.g., de la Beaujardiere et al., 1991; Rich and Hairston, 1994). These measurements, however, were usually limited to specific MLT sectors. For example, some DMSP satellites cross the high-latitude ionosphere roughly along the dawn-dusk line (e.g., Chen et al., 2015). For this reason, merging of data from various time sectors was performed to infer the CRB location for many MLT sectors. The CRB location determination has been simplified with the introduction of the SuperDARN radars whose joint field of view covers a wide region in the MLT-MLAT plane (although not the entire plane). An excellent example of CRB study with SuperDARN is a recent paper by Bristow and Spaleta (2013).

This paper is aimed at a further investigation of the CRB location, in terms of the IMF $B_{z}$ and $B_{y}$, with a goal to infer long-term trends in terms of the season and solar cycle phase.
We use an extensive SuperDARN database for 19 years of operation that we analyze with a non-standard approach of making monthly averaged convection maps.

\section{Approach to analysis}

With the SuperDARN radars, the Potential Fit technique by Ruohoniemi and Baker (1998) and Shepherd and Ruohoniemi (2000) is traditionally used to infer large-scale convection patterns from line-of-sight (LOS) velocity data of multiple radars. Two-minute data sets are usually considered. Bristow and Spaleta (2013) analyzed these kind of maps and inferred the average CRB location by selecting only MLT sectors with good data coverage and then binning the data according to conditions in the solar wind.

In the Potential Fit technique the fitting is done by first representing the electrostatic potential $\left(\Phi_{\mathrm{E}}\right)$ on a thin spherical shell, representing the ionosphere, by a series expansion in terms of spherical harmonic basis functions

$\Phi_{\mathrm{E}}(\theta, \phi)=\sum_{k=0}^{K} \sum_{m=0}^{k} P_{k}^{m}(\cos \theta)\left[g_{k m} \cos m \phi+h_{k m} \sin m \phi\right]$,

where $\theta$ is co-latitude $\left(90^{\circ}\right.$-MLAT), $\phi$ is longitude $\left(\mathrm{MLT} \times 15^{\circ}\right)$, and $P_{k}^{m}(\cos \theta)$ are the associated Legendre functions of order $m$ and degree $k$. Values $g_{k m}$ and $h_{k m}$ are constant fitting coefficients for each combination of $k$ and $m$. $\Phi_{\mathrm{E}}$ is related to the electric field $(E)$ and plasma velocity $(V)$ through

$\boldsymbol{E}=-\nabla \Phi_{\mathrm{E}} ; \quad V=\frac{\boldsymbol{E} \times \boldsymbol{B}}{|\boldsymbol{B}|^{2}}$,

where $\boldsymbol{B}$ is the magnetic field given by the International Geomagnetic Reference Field (IGRF). Fitting coefficients $g_{k m}$ and $h_{k m}$ are determined by minimizing the difference between the observed velocities and the LOS component of the velocities predicted with the above equations. Once $g_{k m}$ and $h_{k m}$ are known, $\Phi_{\mathrm{E}}, \boldsymbol{E}$, and $V$ can be determined at any point in the mapping region (in this case, the high-latitude region where the SuperDARN radars are preforming their measurements).

The spherical harmonic basis functions used by the Potential FIT technique require that observations be distributed about the entire spherical Earth. However, for the SuperDARN data set, measurements are limited to the highlatitude region poleward of 50-60 MLAT. To handle this inconsistency, the SuperDARN data are cleverly redistributed about the entire sphere by multiplying the co-latitude of measurement coordinates by a factor of $180^{\circ} / \theta_{\mathrm{FIT}}$, where $\theta_{\text {FIT }}$ represents the low-latitude boundary of the mapped region. As a consequence of this redistribution, the entire lowlatitude boundary of the mapped region is assigned to a single point at the southern pole, thereby forcing the convection at the boundary of the plot to be a constant value, which is subsequently set to zero. Flow is therefore forced to be 
closed and to be azimuthally directed at the lowest latitudes of the mapped region. Additional constrains are applied to shape the convection pattern following Heppner and Maynard (1987) who found that the convection pattern has a rounded shape on the nightside and a compressed shape on the dayside. To reflect these characteristics, the region between the low-latitude circular boundary of the convection zone defined by $\theta_{\text {FIT }}$ and the Heppner and Maynard boundary (HMB) is filled with low-velocity values of $1 \mathrm{~m} \mathrm{~s}^{-1}$ to indicate that there should not be flow in this region. Accurate mapping therefore requires that the HMB be chosen to coincide with the boundary of the convection zone.

In our work, we process the original SuperDARN data differently. Our argument is that the redistribution of data required by the standard SuperDARN convection mapping technique forces the convection to zero at the boundary of the mapping region, which may not accurately reflect lowlatitude flow. If the boundary of the mapping region does not coincide with the true convection zone boundary, then the convection would be forced to zero where it should not be. If too high a latitude is chosen, this would shift the convection zone boundary to higher latitudes both reducing the low-latitude flow and potentially shifting the location of the CRB to higher latitudes.

In addition, the standard SuperDARN Potential Fit technique relies on contributions from statistical model convection patterns to constrain the shape of the output convection pattern where there are gaps in the data (Shepherd and Ruohoniemi, 2000). Mori and Koustov (2013) showed that the SuperDARN cross polar cap potential (CPCP) deviates noticeably from that given by the statistical model only when the total number of points on a map becomes larger than $\sim 200$; maps with < 200 points are poorly constrained and tend to mimic the statistical model. Summer maps have particularly decreased coverage making the appropriateness of the standard approach for investigation of the seasonal variations questionable. In addition, the SuperDARN radars have poorer echo occurrence rates during solar cycle minimum (Ghezelbash et al., 2014) and the approach of averaging multiple standard SuperDARN maps, each of which might be constrained to follow a statistical model, is vulnerable if the solar cycle effect is to be studied.

To diminish the above weaknesses of the conventional approach by Ruohoniemi and Baker (1998), we decided to consider month-long SuperDARN data sets and infer averaged patterns by using very coarse IMF bins. We first averaged the standard gridded LOS velocities at each MLT-MLAT node (Ruohoniemi and Baker, 1998) over each month of observations. Nodes are uniformly distributed across the highlatitude region with a spacing equivalent to $1^{\circ}$ of MLAT. Convection maps were then generated by fitting the averaged gridded data using the spherical cap harmonic analysis (SCHA) method by Fiori et al. (2010).

The SCHA approach acknowledges that the SuperDARN data set is limited to the high-latitude region which popu-
Table 1. IMF parameter limits selected for producing SuperDARNbased monthly convection maps. The third row shows the low and high limits of the $B_{t}^{-}$bins used.

\begin{tabular}{lcccccc}
\hline & A & B & C & D & E & F \\
\hline$B_{z}$ & - & - & - & - & - & Any \\
$B_{y}$ & Any & Any & Any & - & + & Any \\
$B_{t}, \mathrm{nT}$ & {$[-4,0]$} & {$[-6,-4]$} & {$[-12,-6]$} & {$[-4,0]$} & {$[-4,0]$} & Any \\
\hline
\end{tabular}

lates only a portion of the spherical shell used to represent the ionosphere (i.e., a spherical cap). The electrostatic potential is again represented by a series expansion but this time by using spherical cap harmonic basis functions (Haines, 1985, 2007). Electrostatic potential is represented using the same equation (1), except that the integer value degree $k$ is replaced with a non-integer value $n_{k}(m)$. Fiori et al. (2010) provides a full description of how the SCHA technique can be applied to fit SuperDARN data. The key feature of the SCHA technique is that convection is allowed to cross the boundary of the mapping region (i.e., the region populated by measurements) as it is not forced to zero at the HMB. The disadvantage is that the method sometimes produces unphysical solutions of the numerical fit. For a densely populated map, such features are isolated to the most equatorward regions of the spherical cap over which convection is mapped, well equatorward of the typical location of the CRB. For SuperDARN measurements collected over a 1-month interval, data coverage is more than adequate for reliable convection mapping using SCHA.

We created six data sets so that various conditions in the IMF can be considered for all months. Only negative IMF $B_{z}$ values were considered to deal with 2 -celled patterns and avoid effects of dayside reverse convection cells (or strongly distorted dayside patterns) for positive $B_{z}$ (Cowley and Lockwood, 1992). Table 1 lists the IMF bins used for generation of the data sets. Here and below we use the following notation for the transverse component of the IMF $B_{t}^{-}=\frac{B_{z}}{\left|B_{z}\right|} \cdot \sqrt{B_{z}^{2}+B_{y}^{2}}$, where $B_{z}$ and $B_{y}$ correspond to the north-south and east-west components of the IMF in the GSM coordinate system, and the minus sign reminds the reader that data are for the negative IMF $B_{z}$ component. Bins of $B_{t}^{-}$were selected according to the model of Ruohoniemi and Greenwald (1996) that we will refer to as the RG-96 model. In the rest of the paper, the $B_{t}^{-}$bins are given as ranges denoted as $[a, b]$, where $a$ and $b$ represent the minimum and maximum values of the range. Such binning was chosen so that the CRB determined here could be compared to that of the RG-96 model. The IMF values, necessary for selecting of appropriate bin, were taken from the OMNI database (http://nssdc.gsfc.nasa.gov/omniweb/).

Within each data set, further data binning was done as follows. At each grid point, the data were placed into bins according to the azimuth of the radar beams and/or direction 
(counted in degrees clockwise from magnetic north) of LOS velocity measurement. Azimuth bins were $10^{\circ}$ wide spanning from -180 to $180^{\circ}$ (following the approach of Pettigrew et al., 2010). The average velocity for each azimuthal bin was then determined. Each grid point therefore had up to 36 velocities assigned to it. A SCHA fit was performed to create a map from data selected for each month. The fit was determined based on a spherical cap centered over the North Magnetic Pole having an angular radius of $30^{\circ}$ (spanning from 60 to $90^{\circ}$ of magnetic latitude for all MLT sectors). The procedure was thus very similar to the one recently employed by Cousins and Shepherd (2010), except that they considered multiple bins of the convection drivers and used the standard FIT technique for data processing. We note that SCHA fits appeared to be consistent across the majority of the spherical caps. For maps having a limited amount of data at the most equatorward latitudes, within $\sim 5^{\circ}$ of the lowlatitude boundary of the spherical cap, the fit was not always well constrained. For this reason, we focus on high-latitude velocities located poleward of $70^{\circ}$, well within the polar cap.

In this study, SuperDARN data from 1995 to 2013 (228 months) in the Northern Hemisphere are considered. Figure 1 illustrates background information on the solar activity levels and radar data coverage over the period considered. Figure 1a shows the sunspot number (taken from http: //www.sidc.be/silso/datafiles) and monthly average magnitude of the $B_{t}^{-}$obtained by making histogram distribution for all values over a month and finding the mean value of the histogram, similar to Koustov et al. (2015). The period includes two solar maxima (2000-2002, solar cycle 23 and 20122013, solar cycle 24) and two solar cycle minima (19951998, solar cycle 23 and 2007-2009, solar cycle 24). Solar cycle 23 was strong while solar cycle 24 was weak. The $B_{t}^{-}$ magnitudes are clearly increased during high solar activity and typical values are larger during the maximum of solar cycle 23. Interestingly enough, as the solar cycle progresses from minimum to maximum, $B_{t}^{-}$shows an increasing trend which stops around solar cycle maximum, at which point $B_{t}^{-}$fluctuates significantly, from year to year, around 5-6nT (1999-2004) and 3-5 nT (2010-2013).

As the goal of this study is to characterize the solar cycle and seasonal variations in the CRB location, one concern is data homogeneity over the entire period. It is established that the SuperDARN coverage experiences significant variations (Ghezelbash et al., 2014). This is in part due to a steady increase in the number of operating radars and in part due to varying solar activity changing conditions for the echo onset (Koustov et al., 2014). Figure 1b shows variations in the number of points per standard SuperDARN 2 min convection map, thick black line and scale on the left side of the panel. This number was computed for every month between 1995 and 2013 from standard SuperDARN 2 min grid files. This number is below 100 for periods of low solar activity but rises to $\sim 500$ during the solar cycle 23 maximum years when strong seasonal variations, with much larger num-

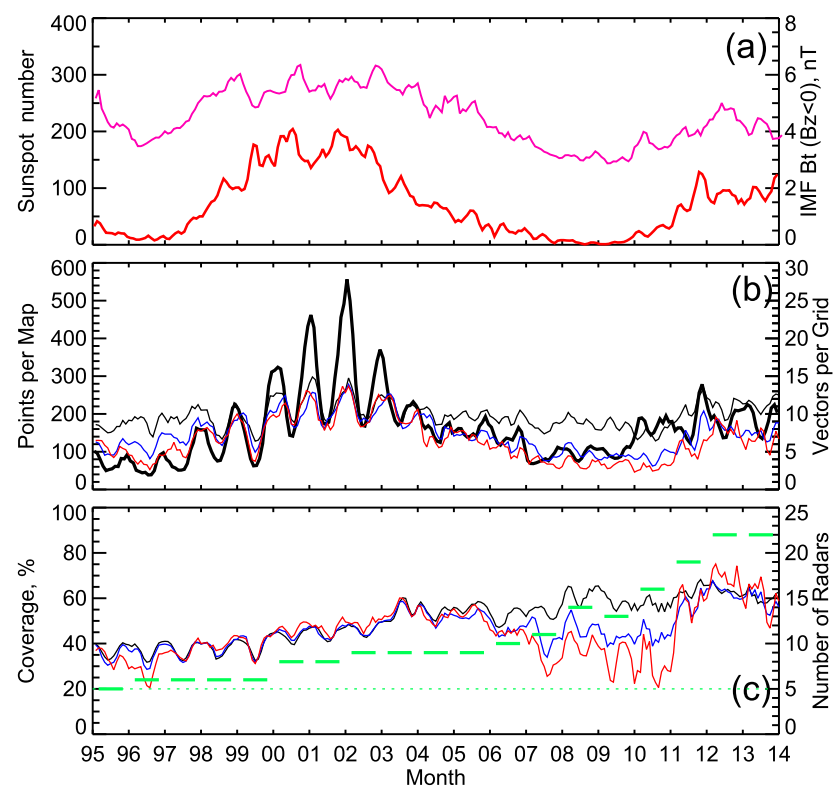

Figure 1. (a) Sunspot number (red) and transverse component of the IMF $B_{t}^{-}$(pink) computed as the average of monthly histogram distributions for $B_{t}^{-}=[-12,0]$ nT. (b) Monthly average number of grid points on the standard 2 min SuperDARN map (thick solid line) and monthly average number of vectors for each grid point. (c) Grid point coverage of the month-long data set at magnetic latitudes poleward of $50^{\circ}$ and all magnetic local times. Thin black, blue and red lines in (b) and (c) correspond to cases A, B, and C (Table 1), respectively.

bers in winter, are noticeable. Seasonal variations are much weaker during the solar cycle 24 maximum years. We note that standard SuperDARN convection maps with the number of points below 200 are hypothesized to be strongly affected by the startup convection model, as detectable through computed cross polar cap potential (Mori and Koustov, 2013). Figure $1 \mathrm{~b}$ thus hints that many SuperDARN maps are, very likely, influenced by the startup convection model, particularly during periods of solar minimum.

Figure $1 \mathrm{~b}$ also shows a parameter that characterizes data reliability in the SCHA method. As explained above, in generating the monthly data set, average vectors are created at each grid point for 36 different possible pointing directions. Additional lines in Fig. 1b represent the average number of vectors determined at each grid point (maximum possible value of 36). Thin black, blue and red lines represent this parameter for the smallest, medium and largest $B_{t}^{-}$bin of the RG-96 model (the scale for these curves is on the right hand side of the panel). We note that this parameter reflects the quality of the SCHA mapping only partially as overall coverage over the map is also important. This parameter shows smallest variations for the smallest $B_{t}^{-}$gate (case A, Table 1) implying that every vector on the data grid is based on about the same database. Figure $1 \mathrm{~b}$ shows that data quality at individual grid points deteriorates during low solar activity for 
the middle and largest bin of the RG-96 model. This is consistent with the decrease in the number of points per standard SuperDARN 2 min map, thick black line (these maps have not been considered in this study). While considering temporal variations of the number of grid points per $2 \mathrm{~min}$ SuperDARN convection map (important for the FIT method) and the number of vectors per grid point on a monthly averaged map (that we consider in our SCHA approach), we found that they correlate reasonably well, the correlation coefficients are $0.7-0.75$ depending on the RG-96 bin.

Figure 1c illustrates the variation of the overall SuperDARN data coverage, which is another important parameter for our SCHA analysis. Here we show the number of grid points (poleward of $50^{\circ} \mathrm{MLAT}$ ) populated by at least one vector divided by the total number of possible grid points, in percent. The plot also shows the number of radars contributing to the data in the Northern Hemisphere, green bars and the scale on the right. A sharp increase in a number of radars occurred after 2010, with the introduction of the middlelatitude SuperDARN radars in the North American sector, is seen. The thin black line in Fig. 1c, corresponding to case A (Table 1), shows an increasing trend; the curve illustrates the advantage of having more radars in the network, at least for the purpose of our analysis. We note that for cases B and C (Table 1), the coverage was depressed in 2006-2010 despite the increased number of radars as compared to the previous solar cycle minimum years of 1995-1998.

Figure 2a gives an example of an averaged convection pattern inferred with our SCHA approach for September 2001 and the IMF bin of $B_{t}^{-}=[-4,0] \mathrm{nT}$ (case A, Table 1). On the map of Fig. 2a, each dot corresponds to a grid point at which velocity data were available. Each dot is the origin of a velocity vector corresponding to the calculated convection pattern. Velocity magnitude is indicated by color. The black contours in Fig. 2a are equipotential lines spaced $6 \mathrm{kV}$ apart with the difference between the maximum and minimum values of the potential (CPCP) of $47.7 \mathrm{kV}$. Velocity vectors follow the equipotential contours. Note that only the portion of the equipotential field lines and velocity vectors located within the spherical maps are plotted.

Several features are seen in Fig. 2a. The pattern has a clear 2-celled structure, as expected for $B_{z}<0$ conditions. The dusk cell is slightly larger than the dawn cell and there is a minor shift of the antisunward polar cap flow toward dawn, away from the noon-midnight meridian. The equipotential contours indicate that the pattern foci are located at MLAT $\sim 75^{\circ}$ and 06:00 and 18:00 MLT. The plasma flow is anti-sunward within the central polar cap. Two major areas of enhanced flows are evident: return plasma flow toward noon during the afternoon hours at MLAT $\sim 70^{\circ}$, and anti-sunward flow over the North Magnetic Pole at MLAT $>80^{\circ}$ during prenoon-dawn hours. The convection reversal boundary is identifiable as an arc-like region of low velocity bounded by flows with opposite directions at MLAT $\sim 75^{\circ}$ between 17:00 and 19:00 MLT and at MLAT $\sim 76^{\circ}$ at 05:00-07:00 MLT.

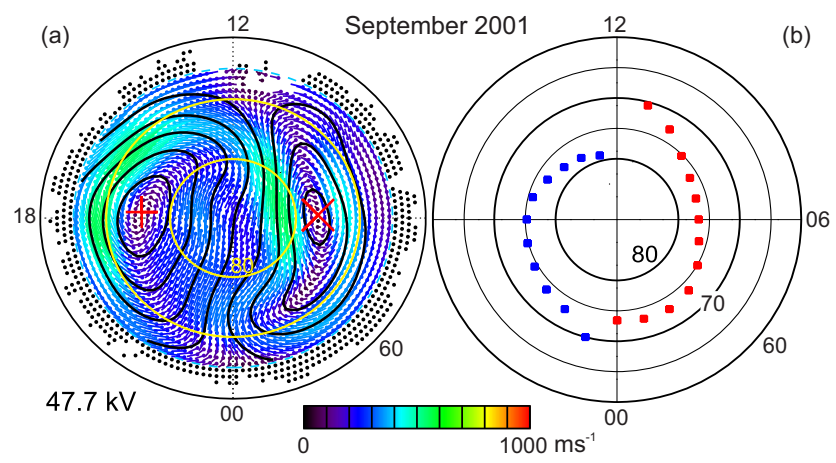

Figure 2. (a) Convection pattern in the MLT-MLAT coordinates inferred from the September 2001 SuperDARN grid velocity data set $\left(B_{t}^{-}=[-4,0] \mathrm{nT}\right)$ by applying the SCHA technique of Fiori et al. (2010). Every dot represents the origin of a plasma velocity vector with the length coded by color. Overlaid are contours of the equal electric potential (that are $6 \mathrm{kV}$ apart). Potential difference between the foci of the large-scale convection cells is $47.7 \mathrm{kV}$. (b) The convection reversal boundary location inferred from the data presented in panel (a) for dusk (blue dots) and dawn (red dots).

To characterize the CRB location quantitatively, we considered velocity data at various latitudes (within a grid having a spacing of $1^{\circ}$ MLAT) averaged over intervals of 1 hour of MLT. We plotted the azimuthal component of the computed flow velocity versus magnetic latitude and identified the intersection of the line with the zero velocity line. Figure $2 b$ gives the inferred locations of the CRB for data in Fig. 2a with dusk and dawn sector data shown in blue and red, respectively. Several features of Fig. $2 b$ are of note. First, the CRB is at about the same latitudes for dusk (18:00 MLT) and dawn (06:00 MLT), 75 and $76^{\circ}$, respectively. For the evening-midnight-morning sector, between 19:00 and 05:00 MLT, the CRB locations are at increasingly lower latitudes approaching midnight. Latitudes separated by $12 \mathrm{~h}$ of MLT (e.g. 19:00 and 05:00 MLT; 20:00 and 04:00 MLT) are similar. The difference in latitude is more obvious for 23:00 and 01:00 MLT. For the prenoon-noonafternoon sector, between 07:00 and 17:00 MLT, the CRB locations are at lower latitudes approaching noon for prenoon data and at higher latitude for afternoon data. This means that there is no "symmetry" between prenoon and afternoon points and there is a jump in the CRB latitude at near noon hours. Chen et al. (2015) referred to this feature as the "gap". The gap in latitudes for points at 11:00 and 13:00 MLT is almost $5^{\circ}$ with prenoon locations being at lower latitudes. For the midnight sector, the (minor) gap in latitudes is also recognizable with the latitudes during post-midnight time to be larger.

We note that for this specific month, it was impossible to determine the CRB location at 12:00 MLT and data are limited in the midnight sector. We have to admit that, in our analysis, derivation of the CRB location at near noon and near midnight is not quite reliable. The highly averaged maps that 
we used show rotational shears with very small latitudinal gradients in the azimuthal flow velocity component in these regions. In addition, gridded velocities are often missed for near noon hours. Although a number for the CRB location was formally derived for many maps, it was often significantly off the trend seen in other MLT sectors. In these cases, the data for near noon (and midnight) observations were excluded.

\section{Effect of the IMF $B_{t}$}

\subsection{Average location}

Data from plots similar to that of Fig. 2b were entered into a database for all 228 months of SuperDARN measurement and, separately, for each bin of the IMF $B_{t}^{-}$(the bins were selected according to Table 1). Fewer data points were present in the spatial bins near midnight and noon MLT. For all the data sets obtained, the average and standard deviation of the MLAT of the CRB were found within each MLT bin. Figure 3 shows inferred data for two $B_{t}^{-}$bins of $[-4,0] \mathrm{nT}$ and $[-12,-6] \mathrm{nT}$, cases $\mathrm{A}$ and $\mathrm{C}$ in Table 1 . These are two extreme RG-96 bins with the smallest and largest $B_{t}^{-}$magnitude.

Figure 3 has the same format as Fig. 2b with the addition of black lines representing the CRB location at \pm standard deviation of latitude. The dotted line shows the CRB location according to Bristow and Spaleta (2013), scaled from their plot in Fig. 7. Their line represents the average CRB location while all possible IMF conditions are considered in their data set. Crosses in Fig. 3a represent the CRB location according to the RG-96 model for the same $B_{t}^{-}$bin. Both plots in Fig. 3 show features seen in Fig. 2b. These are (1) the symmetry in the CRB location for the evening and morning hours (matched MLT sectors such as 19:00 and 05:00 MLT), (2) a minor gap in the MLAT between pre midnight and after midnight locations and (3) an almost $5^{\circ}$ gap in the CRB location at 10:00-12:00 and 13:00-15:00 MLT. A minor difference of Figs. $3 a$ and $2 b$ is that the CRB is at slightly higher MLATs (by $\sim 1^{\circ}$ ) at dusk and dawn. For this $B_{t}^{-}$bin, the CRB locations are at higher latitudes as compared to Bristow and Spaleta (2013) data but reasonably close to those given by the RG-96 model. We note that a difference of only $\sim 1^{\circ}$ must be considered with care, as $1^{\circ}$ is the data resolution used to determine the MLAT of the CRB.

Figure $3 \mathrm{~b}$, for most negative $\operatorname{IMF} B_{t}^{-}$bin, shows that the CRB shifts to lower latitudes for most of MLT sectors. The amount of shift varies, but roughly it is on the order of $2^{\circ}$. Thus the rate of this shift with $B_{t}^{-}$is $\sim 2^{\circ} / 7 \mathrm{nT}$ (middle of the $B_{t}^{-}$bins are -2 and $-9 \mathrm{nT}$ ). The average CRB locations for this $B_{t}^{-}$bin are much closer to the dotted line corresponding to Bristow and Spaleta (2013) data. This is expected since their IMF $B_{z}$ bin is $[-7,-2] \mathrm{nT}$. Another distinct feature of Fig. $3 b$ is that the near noon CRB latitude gap is not very strong. In this paper we do not present data for the mid-

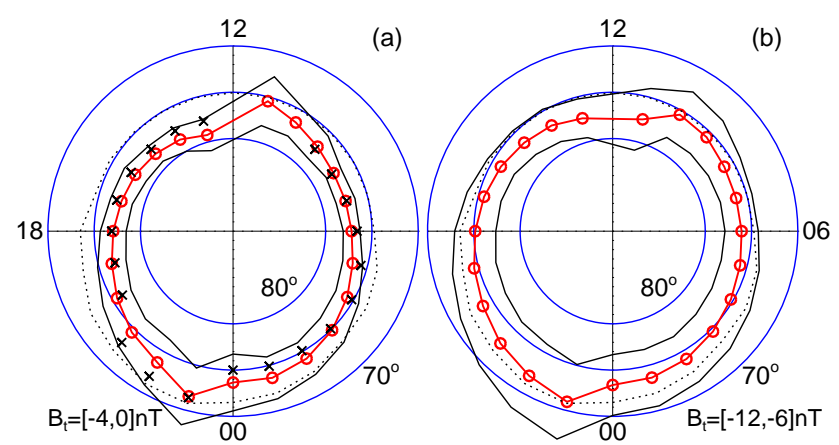

Figure 3. Typical location of the convection reversal boundary (CRB) in the MLT-MLAT coordinates, red dots, for IMF $B_{t}^{-}$in between (a) -4 and $0 \mathrm{nT}$ and (b) -12 and $-6 \mathrm{nT}$. Data for 1995-2013 were considered without binning according to the IMF $B_{y}$. Shown by the solid line is the CRB location \pm 1 standard deviation from the inferred typical location. The dotted line is the CRB location reported by Bristow and Spaleta (2013) for a case of no binning according to the IMF (except considering only $B_{z}<0$ data), scaled from their Fig. 7. Crosses are the CRB location according to the RG-96 model.

dle $B_{t}^{-}$bin of the RG-96 model (case B, Table 1) because the lines on that plot are generally located midway of those shown in Fig. 3a and $\mathrm{b}$ for extreme $B_{t}^{-}$. One can also notice that the dispersion around the mean is larger in Fig. $3 \mathrm{~b}$ for the largest $B_{t}^{-}$magnitudes than in Fig. 3a for the smallest $B_{t}^{-}$magnitudes. This very likely indicates the influence of substorms which are more intense when the magnitude of (negative) $B_{t}^{-}$is larger.

\subsection{Seasonal variations}

Figure 4 plots the sunspot number and CRB location versus month for the entire period under study (1995-2013) and three bins of $B_{t}^{-}$for the dusk sector (between 17:00 and 19:00 MLT). We also present data on the CRB location at dawn, but only for case A to make the diagram cleaner. First consider the dusk data. Variations with large magnitude of up to $\pm 2.5^{\circ}$ are seen between 1995 and 2007 for all three IMF bins with strongest variations for the most negative $B_{t}^{-}$ bin, case $\mathrm{C}$. We will refer to the segment of the data between 1995 and 2007 as period 1, Fig. 4b. Over period 1 there appears to be a shift in the CRB to lower latitudes for more negative $B_{t}^{-}$. This is easy to recognize if one compares the latitude location of the curve and the horizontal dashed line at $77^{\circ}$ MLAT. After 2007, the variations of the CRB location weaken. We will refer to this segment of the data as period 2, see Fig. 4b.

Variations in the CRB latitude during period 1 show a fairly consistent pattern of maxima with highest MLATs achieved during summer and minima with lowest MLATs achieved during winter. The effect is more prominent for case $\mathrm{C}$ (Fig. 4d) where summer-to-winter differences in magnetic latitude are as large as $5^{\circ}$. Also noticeable is the weak- 


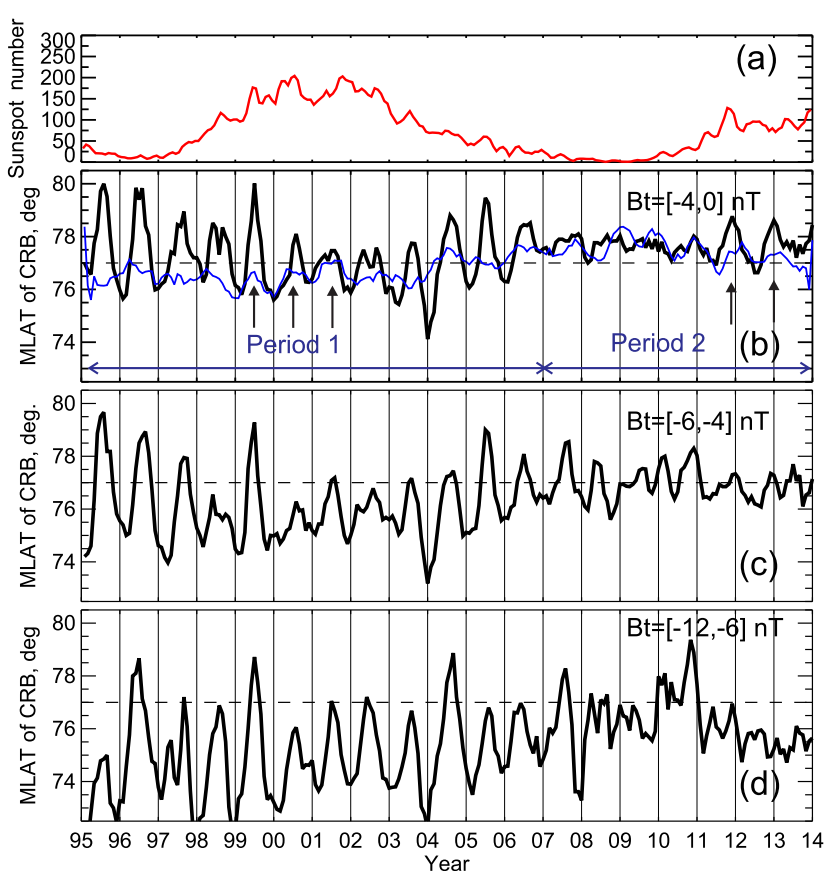

Figure 4. (a) Plot of the Sunspot Number for the period of 19952013 (monthly average number, http://sidc.oma.be/silso). Magnetic latitude of the convection reversal boundary location, black line, at dusk (between 17:00 and 19:00 MLT) for SuperDARN observations between 1995 and 2013 for $B_{z}<0$ and (b) $B_{t}^{-}=[-4,0] \mathrm{nT}$, (c) $B_{t}^{-}=[-6,-4] \mathrm{nT}$ and (d) $B_{t}^{-}=[-12,-6] \mathrm{nT}$, respectively. Panel (b) also shows magnetic latitude of the convection reversal boundary location, blue line, at dawn (between 05:00 and 07:00 MLT). In this panel, arrows indicate some years for which poleward shift of the convection reversal boundary occurs both at dusk and dawn.

ening of the seasonal variations during 2000-2004, which corresponds to high solar activity.

The CRB latitude variations during period 2 do not show a clear pattern. They almost disappear for case A (Fig. 4b) in 2006-2011 but restart in 2011-2013 with winter highest MLATs and summer lowest MLATs. For case B (Fig. 4c), variations are of stronger magnitude with maxima mostly in winter and minima in summer. Data for case C (Fig. 4d) are inconclusive with respect to the seasonal effect as the curve shows more frequent fluctuations.

Figure $4 \mathrm{~b}$ also presents data for the dawn sector (thin blue line) and shows that the magnitude of the latitudinal shift is visibly smaller than for the dusk sector. The pattern of the seasonal changes is less clear as compared to the dusk data. For some years, there is consistency in the CRB location being at higher latitudes both at dawn and dusk in summer so that we can say that there is a "contraction" of the CRB pattern on the plot like that of Fig. 3. These cases occurred during period 1; we indicated several such occasions by arrows in Fig. 4b. For other years of period 1, the seasonal variations of the CRB location at dawn are weak. During pe-

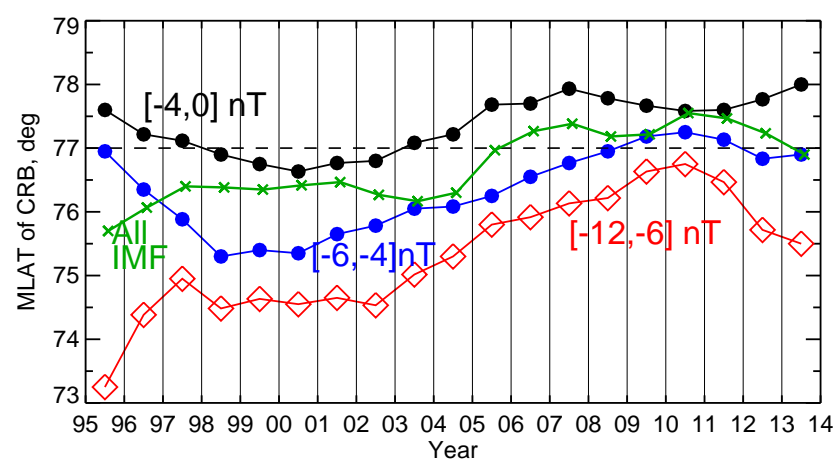

Figure 5. Magnetic latitude of the convection reversal boundary (CRB) location on the dusk side (yearly medians for data between 17:00 and 19:00 MLT) for 19 years of consecutive SuperDARN observations. Presented CRBs are for IMF $B_{z}<0$ and three bins of the $B_{t}^{-}$magnitude: $[-4,0] \mathrm{nT}$ (black dots), [-6,-4] nT (blue dots), and $[-12,-6] \mathrm{nT}$ (red diamonds). Green crosses connected by solid green line are typical CRB locations for a case of all SuperDARN data considered irrespective of the IMF.

riod 2 and only for some years, consistency in the CRB locations at higher latitudes both at dusk and dawn is in winter; these are also indicated by arrows in Fig. 4b. For other IMF bins, cases $\mathrm{B}$ and $\mathrm{C}$, the patterns of the CRB location variations versus season are more complex (respective data are not shown).

\subsection{Solar cycle effect}

Figure $4 \mathrm{~b}$, c hints on the solar cycle trend in the CRB location for cases $\mathrm{B}$ and $\mathrm{C}$ because the curves are well equatorward of $77^{\circ}$ MLAT for period 1 and at roughly $77^{\circ}$ MLAT for period 2. To infer solar cycle variations, we computed yearly average CRB latitudes and placed them on one plot, Fig. 5. Data for all three $B_{t}^{-}$bins show lowest CRB latitudes during the years of high solar activity (1998-2004) and highest latitudes during the years of low solar activity (2006-2010). During 2011-2013 (solar cycle 24 maximum), the data for cases $\mathrm{B}$ and $\mathrm{C}$ with more negative $B_{t}^{-}$show the latitude decrease, which is consistent with the lower latitudes found for the solar cycle 23 maximum, though this does not appear to be the case for case A. We also show in Fig. 5 the yearly average CRB latitudes for case $\mathrm{F}$ for which no binning according to the IMF $B_{t}^{-}$value was considered to produce convection maps (green crosses connected by a green solid line). The curve is positioned in between those for cases A and C, which is not a surprise. The curve shows a tendency for the CRB latitude to be highest during low solar activity of cycle 24 (2006-2011). This effect does not exist during the previous solar cycle minimum of 1995-1998. This result is due to very low CRB latitudes for the largest RG-96 bin (red diamonds); data for two other RG-96 bins do show the highest latitude locations of the CRB in 1995-1998. 


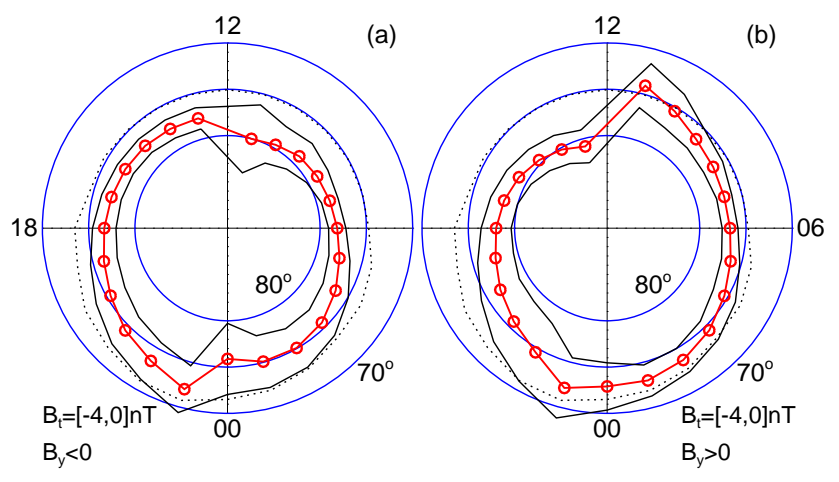

Figure 6. Typical location of the convection reversal boundary (CRB) in the MLT-MLAT coordinates, red circles, for IMF $B_{z}<0$, $B_{t}=[-4,0] \mathrm{nT}$, and (a) IMF $B_{y}<0$ and (b) $B_{y}>0$. Monthly averaged SuperDARN convection maps for 1995-2013 were considered. Shown by the solid line is the CRB location \pm 1 standard deviation from the inferred typical location.

Figures 4 and 5 also indicate that the variations appear to be longer than one solar cycle. This can be seen if one considers the yearly minimum MLAT of the CRB in Fig. 4 or the average MLAT of the CRB over a year in Fig. 5. This is a surprising result and it can be addressed in future work, as the SuperDARN data will be extended to the next solar cycle.

\section{Effect of the IMF $B_{y}$}

\subsection{Average location for $B_{y}>0$ and $B_{y}<0$}

To investigate the influence of the IMF $B_{y}$ on the CRB location, we separated all the data into RG-96 bins according to $B_{t}^{-}$(with $B_{z}$ being always negative) and within each RG-96 bin we considered two large groups, with positive and negative $B_{y}$, Table 1 . We illustrate salient features of the CRB latitude variations (seen in other plots as well) by presenting data for only one RG-96 bin, $B_{t}^{-}=[-4,0]$ nT. Figure 6 shows the inferred CRB latitudes. The format of the presentation here is similar to that of Fig. 3. For Fig. 6, the general look of the patterns is similar to those of Fig. 3. The plots demonstrate two expected effects. First, the "circles" are shifted toward dusk for $B_{y}<0$ and toward dawn for $B_{y}>0$. Second, the latitudinal gaps near noon are such that the CRB location is at higher latitudes during prenoon hours (as compared to afternoon hours) for $B_{y}<0$ and at higher latitudes during afternoon hours (as compared to prenoon hours) for $B_{y}>0$. The latitudinal shift for $B_{y}<0$ is smaller than that for $B_{y}>0$.

Considering plots of Fig. $6 \mathrm{a}, \mathrm{b}$ and comparing with previous publications, several differences can be noted. For the near noon data, the latitudinal gap between the prenoon and afternoon CRB locations is smaller for the $B_{y}<0$ (case D) as compared to reports by Chen et al. (2015) and Bristow and Spaleta (2013). For $B_{y}>0$ (case E), our data show that the CRB location is at higher latitudes during afternoon hours, and the magnitude of the latitudinal gap is comparable to

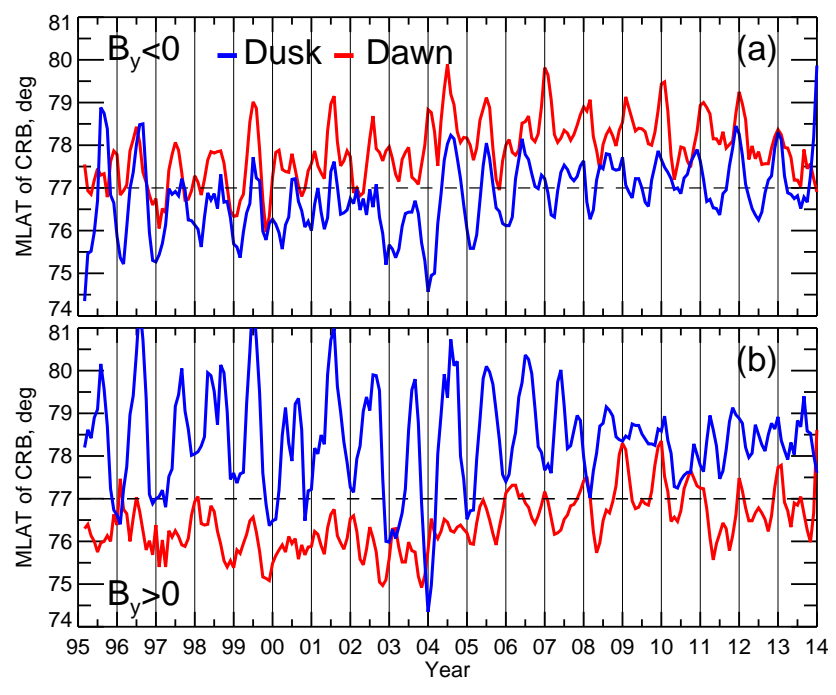

Figure 7. Magnetic latitudes of the convection reversal boundary for (a) $B_{y}<0$ and (b) $B_{y}>0$ (IMF $B_{z}<0$ in both cases, $\left.B_{t}^{-}=[-4,0] \mathrm{nT}\right)$. Monthly averaged SuperDARN convection maps for 1995-2013 were considered. The horizontal dashed line is the latitude of $77^{\circ}$ given for convenience of viewing.

those of Bristow and Spaleta (2013) and Chen et al. (2015). We note that the near noon data for $B_{y}>0$ case are consistent with what we showed in Fig. 3, where no data binning according to the sign of $B_{y}$ was applied, and one can think of these data as corresponding to the case of $B_{y}=0$.

In the midnight sector, the gap in the CRB latitude is strong for case $\mathrm{D}\left(B_{y}<0\right)$; it is $\sim 5^{\circ}$ with the early morning CRB located at higher latitudes. For case $\mathrm{E}\left(B_{y}>0\right)$, the gap is very small. Comparing the CRB curves of the left-hand side (between 13:00 and 23:00 MLT, duskside) for the $B_{y}<0$ and $B_{y}>0$ cases with the CRB curves on the right-hand side (dawnside) of each panel, we can conclude that there is much more similarity in overall shape of the curves for the duskside plots than for the dawnside plots implying that the $B_{y}$ effect is stronger on the dawnside where $B_{y}$-related differences in the CRB latitude can be up to $\sim 5^{\circ}$ during prenoon and early morning hours.

\subsection{Seasonal changes}

Figure 7 presents data for the CRB location at dusk and dawn during 1995-2013 as a line plot. Here the above-mentioned duskward and dawnward shifts of the CRB locations ("circle") are explicit. For $B_{y}<0$ (Fig. 7a), the duskside CRB (blue line) is at $\sim 1.5^{\circ}$ lower latitude, on average. For $B_{y}>0$, case E (Fig. 7b), the dawnside CRB (red line) is at lower latitudes, on average by a larger amount of $\sim 2.5^{\circ}$.

Seasonal variations in the CRB location are obvious in Fig. 7, but their pattern and/or trend is not very consistent over the entire period. The most striking feature is a strong poleward shift in the dusk CRB during summer months of 


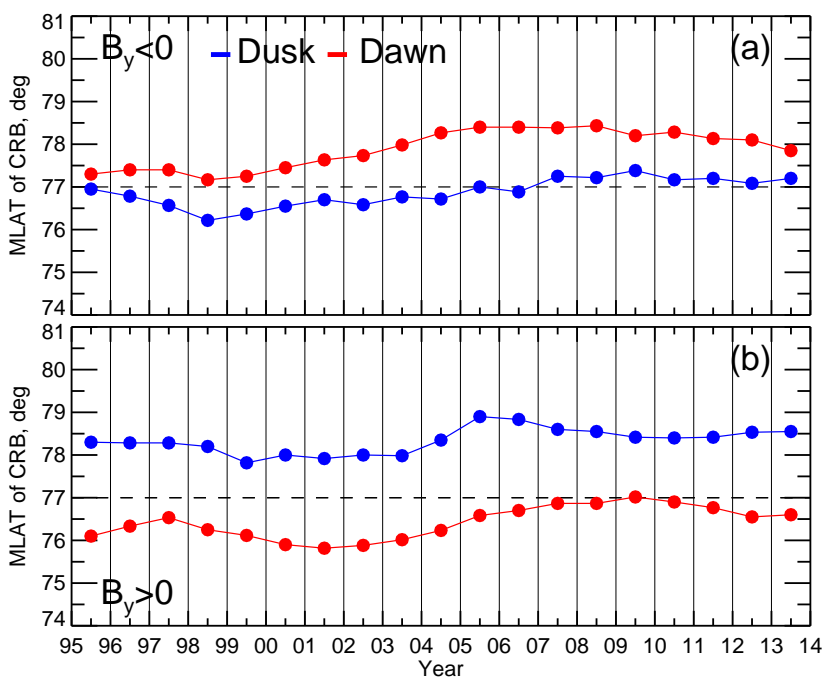

Figure 8. Magnetic latitude of the convection reversal boundary location (yearly medians) on the duskside (between 17:00 and 19:00 MLT, blue line) and dawnside (between 05:00 and 06:00 MLT, red line) for IMF $B_{t}^{-}=[-4,0] \mathrm{nT}$ and (a) $B_{y}<0$ and (b) $B_{y}>0$. The horizontal dashed line is the latitude of $77^{\circ}$ given for convenience of viewing.

1995-2008 (period 1) for the case of $B_{y}>0$ (blue line in Fig. 7b). The magnitude of the shifts decreases drastically after 2008, period 2, and the preferential season for highest latitude occurrence is difficult to discern. Similar shifts of the dusk CRB (of somewhat smaller magnitude) are seen for the case of $B_{y}<0$ (Fig. 7a, blue line) during period 1 and the highest latitudes are achieved more or less consistently in winter during period 2. Compared with the dusk CRB location, the dawn CRB location (red lines in Fig. 7) changes more frequently over a year for some periods (e.g., in 19951997 and 2001-2004) and the magnitude of seasonal shifts (span of latitudes) is smaller. For the dawn data, the highest latitudes are more frequently achieved in summer during period 1 and in winter during period 2 .

\subsection{Solar cycle effect}

Figure 8 presents the yearly median values for the CRB location for cases $\mathrm{D}$ and $\mathrm{E}$ (Table 1) considered in the previous section. Figure 8 simply represents the trends of the data shown in Fig. 7. The dusk data for both cases of $B_{y}<0$ and $B_{y}>0$ (blue lines), show $\sim 0.5^{\circ}$ lower latitudes during high solar activity. This is a remarkably minor effect compared to very strong seasonal variations of the CRB location seen in Fig. 7. The dawn data for both cases of $B_{y}<0$ and $B_{y}>0$ (red lines), show $\sim 1^{\circ}$ lower latitudes during high solar activity which is twice as large as the solar cycle variation seen in the dusk data. The solar cycle effect related to the IMF $B_{y}$ is weaker as compared to that related to the IMF $B_{t}^{-}$(mostly $B_{z}$ controlled), compare Figs. 5 and 8.

\section{Discussion and conclusions}

Previous publications by de la Beaujardiere et al. (1991), Rash et al. (1999), Sotirelis et al. (2005), Bristow and Spaleta (2013) and Chen et al. (2015) established that the CRB location is strongly controlled by the IMF $B_{z}$ and $B_{y}$ components. Recognizing this, we attempted in this study to infer long-term trends in the CRB location by using three bins of the IMF $B_{t}^{-}$and focusing on seasonal and solar cycle variations. The bins were selected to match the RG-96 statistical model bins.

Initially, data were not split according to the sign of IMF $B_{y}$ and the effect of the increase in IMF transverse component $B_{t}^{-}$was investigated while considering only $B_{z}<0$ data. Although both IMF $B_{z}$ and $B_{y}$ affect the CRB location, our idea was to investigate the overall trend without separating these factors. The expectation was that changes in $B_{t}^{-}$would be attributed to changes in $B_{z}$ because over long periods of time, the average $B_{y}$ value is around 0 (see, for example, Fig. 1 in Bristow and Spaleta, 2013). Coarse binning by $B_{t}^{-}$values gave us the chance to separate data into bins with significantly different convection driver magnitudes while maintaining good data coverage.

Data of Fig. 5 showed the CRB shifts toward lower latitudes during periods of high solar activity for case $\mathrm{F}$ (no IMF filtering) by $\sim 1.5^{\circ}$. This effect is expected because $B_{t}^{-}$ values become more negative with the approach of the solar maximum (data of our Figure 1 confirm this for the case of all $B_{t}^{-}$conditions considered). From Figure 1 we estimate that the difference in the typical $B_{t}^{-}$values in 2001 (solar maximum) and 2008 (solar minimum) is $\sim 2 \mathrm{nT}$. Thus, based on the solar cycle trend data, the CRB equatorward shift is on the order of $\sim 1.5^{\circ} / 2 \mathrm{nT}$.

Another quantitative assessment of the $B_{t}^{-}$effect, in the dusk sector, is performed in Fig. 9 where we plot the monthly average CRB location versus the monthly average $B_{t}^{-}$value (shown in Fig. 1a). Data in all three cases of the RG-96 model are considered (cases A, B and C, Table 1). The point clustering around $B_{t}^{-}=-2 \mathrm{nT},-5 \mathrm{nT}$ and $-8 \mathrm{nT}$ is obvious. Because of the large data spread within each RG-96 band, we binned the data with a step of $0.3 \mathrm{nT}$ and then made linear fits to binned values in each band. The slopes and $y$-intercepts of the inferred lines are presented in Table 2. Slopes in Table 2 imply that the rate of the CRB equatorward shift is (1.5$\left.2^{\circ}\right) / 2 \mathrm{nT}$, consistent with our estimate from the solar cycle effect (we believe that case B is not representative because the bin of $B_{t}^{-}$is too small to assess the slope properly). We note that the slopes presented for cases A, B, and C, were determined for limited data sets (over 3-7 bins/red dots in Fig. 9) that might not necessarily be representative of an overall trend. In Fig. 9 we also made a linear fit to all small diamonds, reflecting all the data available for 228 months. The slope here is somewhat smaller, $\sim 1^{\circ} / 2 \mathrm{nT}$. Our estimate of the CRB latitude response to a change in the IMF $B_{t}^{-}$of (1.5$\left.2^{\circ}\right) / 2 \mathrm{nT}$ is reasonably consistent with the results of Bristow 


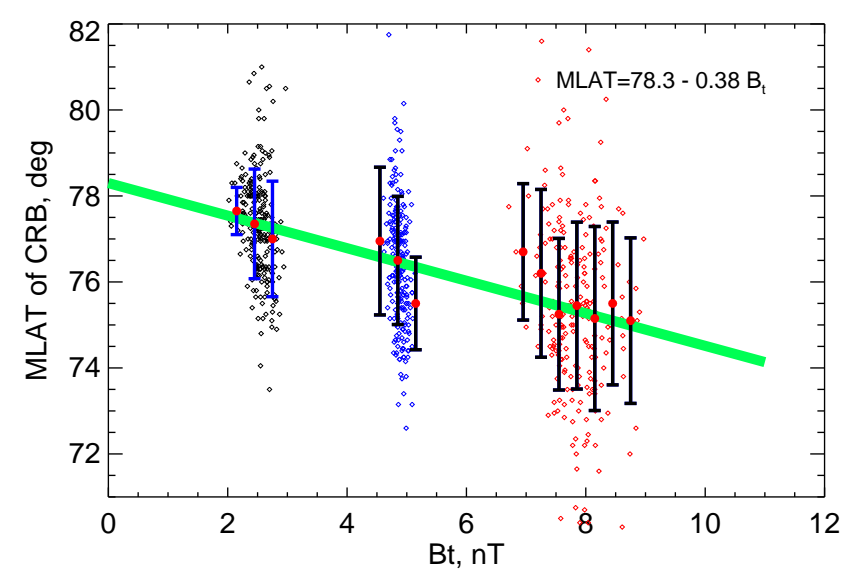

Figure 9. MLAT of the convection reversal boundary location in the dusk sector. Black, blue and red points (small diamonds) correspond to the RG-96 model bands, cases A, B and C (Table 1), respectively. Large red circles are the binned values of the magnetic latitude made with a step of $0.3 \mathrm{nT}$. Vertical bars are the median value of the $\mathrm{CRB}$ location within each bin \pm one standard deviation from the median value. The green line is the best linear fit line to all the points. The $y$-intercept and the slope of the line are shown in the upper right corner.

Table 2. $Y$-intercept and slope of the linear fit to the MLAT- $B_{t}^{-}$ dependence for a number of $B_{t}^{-}$bands as described in Table 1. Data in the three left columns characterize the fits within specific RG-96 model bands (in Fig. 9, fits to red dots within each RG-96 model band). Data in the three right columns are obtained by fitting to all available points (in Fig. 9, black, blue and red small diamonds).

\begin{tabular}{lcccccc}
\hline & $\mathrm{A}$ & $\mathrm{B}$ & $\mathrm{C}$ & All & Summer & Winter \\
\hline Intercept, $^{\circ}$ & 79.8 & 87.7 & 81.4 & 78.3 & 78.8 & 78.2 \\
Slope, $^{\circ} / \mathrm{nT}$ & -1.1 & -2.41 & -0.75 & -0.38 & -0.29 & -0.48 \\
\hline
\end{tabular}

and Spaleta (2013) and Chen et al. (2015). This value corresponds to a stronger dependence that we estimated from our Fig. $3\left(\sim 2^{\circ} / 7 \mathrm{nT}\right)$. We note that Fig. 3 is based on all the data available, including years of the low and high solar activity, and thus the found difference is not a surprise.

Data of Fig. 5 for individual bins of the IMF $B_{t}^{-}$also showed the CRB shifts equatorward over the solar cycle and, remarkably, of a comparable magnitude, $\sim 2^{\circ}$ for case $\mathrm{C}$ and $\sim 1-1.5^{\circ}$ for cases $\mathrm{A}$ and $\mathrm{B}$. We inferred a comparable value for the dawnside CRB (for example, see Fig. 4b, blue line or other data presented in Abooalizadeh, 2015). These shifts are difficult to explain by variations in the IMF $B_{t}^{-}$. Koustov et al. (2015) presented data on typical $B_{t}^{-}$values for all three cases (their Fig. 2h) showing $\sim 20,18$ and 5\% increases in the $B_{t}^{-}$magnitude toward the solar cycle maximum for cases $\mathrm{A}, \mathrm{B}$ and $\mathrm{C}$, respectively. This is not sufficient to explain the trends in Fig. 5 because for these $B_{t}^{-}$changes, the solar cycle CRB shift should be a fraction of a degree for a $B_{t}^{-}$change of $2 \mathrm{nT}$. The unexpectedly strong solar cycle effect for individual $B_{t}^{-}$bins, seen in Fig 5, suggests that the rate of the equatorward shift of the CRB is affected by other parameters and/or solar wind drivers that we neglected in our analysis. We can, however, conclude that the solar cycle trends reported in our Fig. 5 are, partially, a reflection of the fact that with the solar activity increase, stronger IMF $B_{t}^{-}$occur more frequently even within relatively narrow IMF bins.

We also investigated seasonal variations in the CRB location, our Fig. 4. Several previous publications conclude that the high-latitude convection pattern, including the location of the CRB, show little change with season (e.g., Holt et al., 1987). Unfortunately, most previous studies do not focus on the CRB location. For example, Rich and Hairston (1994) present patterns for three seasons and various IMF conditions. Inspection of their data by eye gives an impression that there are some visible differences in the CRB location, but their quantification is difficult. Similar conclusions can be made from SuperDARN-based plots in Ruohoniemi and Greenwald (2005), their Figs. 5 and 6. In a more focused study, de la Beaujardiere et al. (1991) reported that in the afternoon sector the CRB is at either the same or higher latitudes during the summer. The effect is not very strong. We reviewed our data for individual months and in the afternoon and found consistency with this result. Our data of Fig. 4 for the duskside (period 1) are also consistent with the results of de la Beaujardiere et al. (1991). Moreover, our plots of Fig. 4 for period 1 show that the shifts are larger for stronger IMF $B_{t}^{-}$(and thus more negative $B_{z}$ ) and can be as large as $5^{\circ}$. However, data for period 2 of Fig. 4 are in disaccord with de la Beaujardiere et al. (1991). For the pre-noon hours, de la Beaujardiere et al. (1991) reported that in winter the $\mathrm{CRB}$ is at a higher latitude. Our analysis of the pre-noon data and dawn sector data (not presented in this paper) showed inconsistency with this prediction for period 1 and consistency during period 2. Reasons for a change in the pattern of seasonal dependence for the CRB location and weakening of the summer-winter contrast after $\sim 2008$ in our data are not clear. Our original thought was that the seasonal variations in the CRB location are due to a poleward shift of summer field-aligned currents (e.g., Green et al. (2009) and references therein). But this effect would not explain our data for period 2 although we do not know if the FAC seasonal shifts are still in effect (recent FAC data have not been statistically investigated, to the best of our knowledge).

In our second step we split the data for cases of $B_{y}>0$ and $B_{y}<0$ for the smallest RG-96 IMF bin of $B_{t}^{-}=[-4,0] \mathrm{nT}$. In view of very recent results by Chen et al. (2015), this is certainly too course a binning for investigating the $B_{y}$ effect. However, it is a reasonable first step in assessing the trends.

Data in Fig. 6 confirm previous reports of the dawndusk asymmetry of the CRB location by Bristow and Spaleta (2013) and Chen et al. (2015). Averaged over all seasons and for $B_{y}<0$, Fig. 6, the dusk CRB shifts to lower latitudes while the dawn CRB shifts to higher latitudes. This 
is consistent with the fact that the dawn cell of the convection pattern is of a round shape with its center located at higher latitudes as compared to the case of $B_{y}=0$. The dusk crescent shape cell is being "squeezed" to lower latitudes so that the CRB on the duskside shifts to lower latitudes. For the $B_{y}>0$ case, the more round dusk cell "expands" toward dawn so that the more crescent-like dawn cell has the CRB at a lower latitude.

Our attempt to infer the CRB seasonal variations due to the $B_{y}$ effect was successful only for the dusk sector. For the case of $B_{y}>0$, the dusk CRB shifts to higher latitudes in summer during period 1 . Interestingly enough, the magnitudes of the shift here are much stronger than in cases $\mathrm{A}-\mathrm{C}$ implying that the IMF $B_{y}$ affects the CRB location at various seasons in a more significant way than the IMF $B_{z}$. This conclusion is only applicable to the near dusk and dawn data. For the $B_{y}<0$ case, Fig. 7a, the dusk data show highest latitudes in summer during period 1 as well and, clearly, in winter during period 2. For the dawn sector, we were unable to infer a consistent pattern in the CRB location change with season. In terms of the solar cycle variation, we found that the poleward CRB shifts during low solar activity periods are weaker while data are binned according to the IMF $B_{y}$ as compared to cases while the data are binned according of IMF $B_{t}^{-}\left(B_{z}\right)$.

We would like to mention three major differences of our results regarding the CRB location on an MLT-MLAT plane with those reported in previous studies. First, our typical latitudes, particularly along the dawn-dusk meridian, are somewhat higher than those reported by Bristow and Spaleta (2013) but comparable to those reported by Chen et al. (2015) and according to the RG-96 model. Data of Sotirelis et al. (2005), their Fig. 6 for 06:00 and 18:00 MLT, appear to be closer to our numbers.

The second issue is with respect to the near noon CRB latitudinal gaps. Chen et al. (2015) found, from DMSP data, that for $B_{y}<0$ case, the CRB gap is stronger for more negative $B_{y}$ and can be as large as $\sim 10^{\circ}$. The CRB gap disappears as $B_{y}$ becomes close to 0 and, for $B_{y}>0$, the gap changes its configuration so that the $\mathrm{CRB}$ latitudes are higher in afternoon (for strong $B_{t}^{-}$, this latter effect is not seen in our data). SuperDARN data by Bristow and Spaleta (2013) also show differences in the CRB latitudes just prior to and after noon, depending on the IMF $B_{y}$ sign. Their data (Fig. 9b) are consistent with the results of Chen et al. (2015) except the magnitude of the gap is smaller for the $B_{y}<0$ case and larger for the case of $B_{y}>0$. Perhaps these differences are owing to the different bins of the IMF $B_{y}$ in these studies. Our data of Fig. 6 are qualitatively consistent with the expected $B_{y}$ effect but definitively only for the $B_{y}>0$. In our case, the near noon CRB gap is on the order of $5^{\circ}$, which is more consistent with conclusions of Bristow and Spaleta (2013) than those of Chen et al. (2015). For $B_{y}<0$, our data show only a minor gap although of the consistent "configuration". Once again, we have to admit that our derivation of the CRB location at near noon and near midnight is not quite reliable and the dif- ferences at near noon and midnight with other publications are not unexpected.

The third issue is with respect to the CRB gap on the nightside. The CRB location in this sector is strongly affected by the substorm activity, e.g. Bristow et al. (2003). Recently, Imber et al. (2013) showed that the Heppner-Maynard (HMB) boundary at midnight, routinely determined in the SuperDARN mapping procedure, reasonably reflects the location of the open-closed magnetic field boundary (OCB). They demonstrated that as the solar drivers, and substorm activity increases, the HMB shifts equatorward, and as the solar drivers and substorm activity decreases, the HMB shifts poleward. Although the relationship between the OCB and CRB locations is complicated in the midnight sector (e.g., Sotirelis et al., 2005), some degree of correlation is expected. In this respect, our pre-midnight data also show the above tendencies; the CRB location during pre-midnight hours is at a lower latitude for larger $\left|B_{t}^{-}\right|$, compare Fig. 3a and b. The effect is also seen in Bristow and Spaleta (2013) data, their Fig. 9a.

We summarize the results of this study as follows. Evaluated in bins of the RG-96 convection model for $B_{z}<0$ :

1. The CRB latitude shows seasonal variations with highest latitude reached during summer for observations in 1995-2007 and during winter for observations in 20082013. Summer-winter differences within individual year can be as large as $5^{\circ}$ for the first period and $2^{\circ}$ for the second period.

2. The seasonal effect is stronger for larger $B_{t}^{-}$values and $B_{y}>0$, in both cases on the duskside. The equatorward shift in the CRB latitude with the $B_{t}^{-}$increase is on the order $\left(1-2^{\circ}\right) / 2 \mathrm{nT}$.

3. On average (over a year), for the same $B_{t}^{-}$bin, the CRB shifts by $1-2^{\circ}$ to lower latitudes during high solar activity.

4. For IMF $B_{y}<0$, on average, the CRB shifts equatorward at dusk and poleward at dawn. For $B_{y}>0$, on average, the CRB shifts poleward at dusk and equatorward at dawn. The shifts in the CRB locations are on the order of 1-2 ${ }^{\circ}$. During 1995-2007, higher dusk CRB latitudes were observed in summer for both $B_{y}<0$ and $B_{y}>0$ cases. During 2008-2013, the highest dusk CRB latitudes were observed in winter for the case of $B_{y}<0$. At dawn, a more consistent pattern of seasonal shifts was seen during 2008-2013 when the highest CRB latitudes were seen in winter, but overall the pattern of seasonal changes is more complicated as compared to the dusk case. 
Acknowledgements. SuperDARN is a collection of radars funded by national scientific agencies of Australia, Canada, China, France, Italy, Japan, South Africa, United Kingdom and United States of America. The research was supported by NSERC Discovery grant to AVK. Work by RADF was supported by the Natural Resources Canada, Earth Sciences Sector, Public Safety Geosciences program and the Canadian Space Agency. We thank K. McWilliams for continuously updating the SuperDARN convection map database at the Saskatoon node and making it available to us. Critical comments by both reviewers are appreciated.

The topical editor, S. Milan, thanks B. Hubert and one anonymous referee for help in evaluating this paper.

\section{References}

Abooalizadeh, Z.: Long-term variations in the high-latitude plasma flows inferred from SuperDARN radar data, M.Sc. thesis, University of Saskatchewan, Saskatoon, Canada, available at: http://hdl.handle.net/10388/ETD-2015-04-2041 (last access: 10 February 2016), 2015.

Bristow, W. A. and Spaleta, J.: An investigation of the characteristics of the convection reversal boundary under southward interplanetary magnetic field, J. Geophys. Res., 118, 6338-6351, 2013.

Bristow, W. A., Sofko, G. J., Stenbaek-Nielsen, H. C., Wei, S., Lummerzheim, D., and Otto, A.: Detailed analysis of substorm observations using SuperDARN, UVI, ground-based magnetometers, and all-sky imagers, J. Geophys. Res., 108, 1124, doi:10.1029/2002JA009242, 2003.

Chen, Y.-J., Heelis, R. A., and Cumnock, A.: Response of the ionospheric convection reversal boundary at high latitudes to changes in the interplanetary magnetic field, J. Geophys. Res.-Space, 120, 5022-5034, doi:10.1002/2015JA021024, 2015.

Cousins, E. D. P. and Shepherd, S. G.: A dynamical model of high-latitude convection derived from SuperDARN plasma drift measurements, J. Geophys. Res., 115, A12329, doi:10.1029/2010JA016017, 2010.

Cowley, S. W. H. and Lockwood, M.: Excitation and decay of solar wind-driven flows in the magnetospere-ionosphere system, Ann. Geophys., 10, 103-115, 1992.

de la Beaujardiere, O., Alcayde, D., Fontanari, J., and Leger, C.: Seasonal dependence of high-latitude electric fields, J. Geophys. Res., 96, 5723-5735, 1991.

Fiori, R. A. D., Boteler, D. H., Koustov, A. V., Haines, G. V., and Ruohoniemi, J. M.: Spherical cap harmonic analysis of Super Dual Auroral Radar Network (SuperDARN) observations for generating maps of ionospheric convection, J. Geophys. Res., 115, A07307, doi:10.1029/2009JA015055, 2010.

Foster, J. C.: An empirical electric field model derived from Chatanika radar data, J. Geophys. Res., 88, 981-987, 1983.

Ghezelbash, M., Fiori, R. A. D., and Koustov, A. V.: Variations in the occurrence of SuperDARN F region echoes, Ann. Geophys., 32, 147-156, doi:10.5194/angeo-32-147-2014, 2014.

Green, D. L., Waters, C. L., Anderson, B. J., and Korth, H.: Seasonal and interplanetary magnetic field dependence of the field-aligned currents for both Northern and Southern Hemispheres, Ann. Geophys., 27, 1701-1715, doi:10.5194/angeo-271701-2009, 2009.
Haines, G.: Encyclopedia of Geomagnetism and Paleomagnetism, chap. Spherical Cap Harmonics, 395-397, Encyclopedia of Earth Sciences, Springer, Dordrecht, the Netherlands, 2007.

Haines, G. V.: Spherical cap harmonic analysis, J. Geophys. Res., 90, 2583-2591, 1985.

Heppner, J. P. and Maynard, N. C.: Empirical high-latitude electric field models, J. Geophys. Res., 92, 4467-4489, 1987.

Holt, J. M., Wand, R. H., Evans, J. V., and Oliver, W. L.: Empirical models for the plasma convection at high latitudes from Millstone Hill observations, J. Geophys. Res., 92, 203-212, 1987.

Hubert, B., Aikio, A. T., Amm, O., Pitkänen, T., Kauristie, K., Milan, S. E., Cowley, S. W. H., and Gérard, J.-C.: Comparison of the open-closed field line boundary location inferred using IMAGEFUV SI12 images and EISCAT radar observations, Ann. Geophys., 28, 883-892, doi:10.5194/angeo-28-883-2010, 2010.

Imber, S. M., Milan, S. E., and Lester, M.: Solar cycle variations in polar cap area measured by the SuerDARN radars, J. Geophys. Res.-Space, 118, 6188-6196, doi:10.1002/jgra.50509, 2013.

Knipp, D. J., Emery, B. A., Richmond, A. D., Crooker, N. U., Hairston, M. R., Cumnock, J. A., Denig, W. F., Rich, F. J., de la Beaujardiere, O., Ruohoniemi, J. M., Rodger, A. S., Crowley, G., Ahn, B.-H., Evans, D. S., Fuller -Rowell, T. J., Friis Christensen, E., Lockwood, M., Kroehl, H. W., Maclennan, C. G., McEwin, A., Pellinen, R. J., Morris, R. J., Burns, G. B., Papitashvili, V., Zaitzev, A., Troshichev, O., Sato, N., Sutcliffe, P., and Tomlinson, L.: Ionospheric convection response to slow, strong variations in a northward interplanetary magnetic field: A case study for January 14, 1988, J. Geophys. Res., 98, 1927319292, doi:10.1029/93JA01010, 1993.

Koustov, A. V., Ponomarenko, P. V., Ghezelbash, M., Themens, D. R., and Jayachandran, P. T.: Electron density and electric field over Resolute Bay and $\mathrm{F}$ region ionospheric echo detection with the Rankin Inlet and Inuvik SuperDARN radars, Radio Sci., 49, 1-12, doi:10.1002/2014RS005579, 2014.

Koustov, A. V., Fiori, R. A. D., and Aliaboozadeh, Z.: Long-term variations in the intensity of polar cap plasma flows inferred from SuperDARN, J. Geophys. Res. Space, 120, 9722-9737, doi:10.1002/2015JA021625, 2015.

Lu, G., Richmond, A. D., Emery, B. A., Reiff, P. H., de la Beaujardière, O., Rich, F. J., Denig, W. F., Kroehl, H. W., Lyons, L. R., Ruohoniemi, J. M., Friis-Christensen, E., Opgenoorth, H., Persson, M. A. L., Lepping, R. P., Rodger, A. S., Hughes, T., McEwin, A., Dennis, S., Morris, R., Burns, G., and Tomlinson, L.: Interhemispheric asymmetry of the high-latitude ionospheric convection pattern, J. Geophys. Res., 99, 6491-6510, 1994.

Mori, D. and Koustov, A. V.: SuperDARN cross polar cap potential dependence on the solar wind conditions and comparisons with models, Adv. Space Res., 52, 1155-1167, 2013.

Papitashvili, V. and Rich, F. J.: High-latitude ionospheric convection models derived from Defense Meteorological Satellite Program ion drift observations and parameterized by the interplanetary magnetic field strength and direction, J. Geophys. Res., 107, 1198, doi:10.1029/2001JA000264, 2002.

Papitashvili, V. O., Belov, B. A., Faermark, D. S., Feldstein, Y. I., Golyshev, S. A., Gromova, L. I., and Levitin, A. E.: Electric potential patterns in the northern and southern polar regions parametrized by the interplanetary magnetic field, J. Geophys. Res., 9, 13251-13262, 1994. 
Pettigrew, E. D., Shepherd, S. G., and Ruohoniemi, J. M.: Climatological patterns of high-latitude convection in the northern and southern hemispheres: Dipole tilt dependencies and interhemispheric comparisons, J. Geophys. Res., 115, A07305, doi:10.1029/2009JA014956, 2010.

Rash, J. P. S., Rodger, A. S., and Pinnock, M.: HF radar observations of the high-latitude ionospheric convection pattern in the morning sector for northward IMF and motion of the convection reversal boundary, J. Geophys. Res., 104, 14857-14866, 1999.

Rich, F. C. and Hairston, M.: Large-scale convection patterns observed by DMSP, J. Geophys. Res., 99, 3827-3844, 1994.

Ridley, A. and Clauer, C. R.: Characterization of the dynamic variations of the dayside high-latitude ionospheric convection reversal boundary and relationship to interplanetary magnetic field orientation, J. Geophys. Res., 101, 10919-10938, 1996.

Ruohoniemi, J. M. and Baker, K. B.: Large-scale imaging of highlatitude convection with SuperDual Auroral Radar network HF radar observation, J. Geophys. Res., 103, 20797-20811, 1998.

Ruohoniemi, J. M. and Greenwald, R. A.: Statistical patterns of high-latitude convection obtained from Goose Bay HF radar observations, J. Geophys. Res., 101, 22743-21763, 1996.
Ruohoniemi, J. M. and Greenwald, R. A.: Dependencies of high-latitude plasma convection: Consideration of interplanetary magnetic field, seasonal, and universal time factors in statistical patterns, J. Geophys. Res., 110, A09204, doi:10.1029/2004JA010815, 2005.

Shepherd, S. G. and Ruohoniemi, J. M.: Electrostatic potential patterns in the high-latitude ionosphere constrained by SuperDARN measurements, J. Geophys. Res., 105, 23005-23014, 2000.

Sotirelis, T., Ruohoniemi, J. M., Barnes, R. J., Newell, P. T., Greenwald, R. A., Skura J. P., and Meng, C.-I.: Comparison of SuperDARN radar boundaries with DMSP particle precipitation boundaries, J. Geophys. Res., 110, A06302, doi:10.1029/2004JA010732, 2005.

Zhang, S.-R., Holt, J. M., and McCready, M.: High latitude convection based on long-term incoherent scatter radar observations in North America, J. Atmos. Sol.-Terr. Phys., 69, 1273-1291, 2007. 